\title{
Testing association and maternally mediated genetic effects with the principal component analysis in case-parents studies
}

\author{
Yumei Li and Yang Xiang
}

\begin{abstract}
s
Background: Major advances in genotyping technology have generated high-density maps of single nucleotide polymorphism (SNP) markers that provide an unprecedented opportunity to identify genes underlying complex traits. Several family-based statistical methods showing robust population stratification have been developed to test the association between multiple markers and disease-susceptibility genes. Only a few methods focus on testing for maternally mediated genetic effects, which is a critical risk for birth defects. The present study focuses on testing for association and maternally mediated genetic effects with family-based methods.

Results: In the present study, we proposed a new method, max_PC integrating principal component analysis, to test association or maternally mediated genetic effects with case-parent data. The proposed method only uses the genotypes of case-parents triads and accommodates missing SNP data. Our results demonstrated that this method is powerful to test association or maternally mediated genetic effects and attractive because it provides a tool for testing the null hypothesis of no association and no maternally mediated genetic effects. Simulations with the permutation procedure as well as an application in the Crohn's disease study showed that the type I error rates of the proposed statistic were nominal with slightly higher power as compared to those of the max $Z^{2}$ test.
\end{abstract}

Conclusions: We conclude that the max_PC is a good approach to test association or maternally mediated genetic effects.

Keywords: Association analysis, Maternally mediated genetic effects, Case-parent triads, Principal component analysis

\section{Background}

The rapid advancement of genotyping technologies and the availability of enormous quantities of genotype or haplotype data provide an unprecedented opportunity for identifying genes involved in susceptibility to complex diseases. The utilization of information from individual markers as well as linkage disequilibrium (LD) structure between the markers has resulted in the use of haplotypebased association studies to determine the relationship between complex traits and a series of possibly linked markers. However, haplotype-based methods are challenging as the large number of distinct haplotypes will in turn produce a large number of degrees of

\footnotetext{
* Correspondence: liym74@yahoo.com

School of Mathematics and Computational Science, Huaihua University, Huaihua, Hunan 418008, P. R. China
}

(c) $2016 \mathrm{Li}$ and Xiang. Open Access This article is distributed under the terms of the Creative Commons Attribution 4.0 International License (http://creativecommons.org/licenses/by/4.0/), which permits unrestricted use, distribution, and reproduction in any medium, provided you give appropriate credit to the original author(s) and the source, provide a link to the Creative Commons license, and indicate if changes were made. The Creative Commons Public Domain Dedication waiver (http://creativecommons.org/publicdomain/zero/1.0/) applies to the data made available in this article, unless otherwise stated. freedom. In addition, some haplotype-based methods need the estimation of haplotype phases when haplotypes are not directly observed. A potential strategy to avoid the estimation of haplotype phases and frequencies is to develop genotype-based statistical approaches that directly use genotype data.

Population-based case-control studies and family-based studies are two common strategies for association analysis. Compared to case-control studies, family-based studies are more attractive due to their robustness to population stratification. Several genotype-based methods with case-parent data have been proposed for association test. McIntyre et al. [1] proposed a max_TDT test, in which the usual TDT statistic for each locus was calculated and the maximum was utilized as the test statistic. Using the principal components $(\mathrm{PC})$ of a variance-covariance matrix of difference vectors that were calculated by comparing the 
genotypes of affected offspring with their corresponding "complements", which are defined to be the parental genotypes that were not present in the affected offspring, Lee WC [2] proposed the adaptive principal component test, $P C T_{L}^{2}$. Based on the same difference vector, Fan et al. [3] proposed a paired Hotelling's $\mathrm{T}^{2}$ test, while Shi et al. [4] developed the max_ $Z^{2}$ test. The max_ $Z^{2}$ test is superior to other methods as it is able to study the maternally mediated genetic effects while only using the genotypes of affected individuals and their parents. It could also accommodate missing SNP data. Maternally mediated genetic effects arise when the phenotype of an offspring is influenced by maternal alleles via the intrauterine environment [5]. This effect can be detected by using the father's genotype as a matched control for the mother's under an assumption of mating symmetry [6]. The maternal genome influences the risk for specific diseases in the offspring, suggesting maternal genes play an independent role in the etiology of birth defects [5, 6]. So incorporating maternally mediated genetic effects into an association analysis might be particularly useful in studying diseases that happened during fetal life.

In this report, we proposed a new statistic, max_PC, to test association and maternally mediated genetic effects with case-parent data. Here, the null hypothesis is that neither association nor maternally mediated genetic effects exist. The max_PC test has three characteristics: (1) only using the genotypes of case-parent triads while accommodating missing SNP data, (2) utilizing the principal component analysis, and (3) evaluating the association and the maternally mediated genetic effects at the same time. Simulations analysis using a permutation procedure and an application in Crohn's disease study indicated that this max_PC statistic has good performance.

\section{Results}

\section{Simulation setting}

To assess the performance of the statistic, $\max _{-} P C$, we performed a simulation study using a wide range of parameters. The simulations were implemented as described by Lee WC [2]. A candidate region of $100 \mathrm{~kb}$ in size was considered, where 20 dense marker loci were uniformly distributed in a homogeneous population. The marker frequencies were randomly determined, with values ranging from $0.1-0.9$, and the disequilibrium coefficients between two adjacent markers were uniformly generated between -0.9 and 0.9 [2, 7]. Assumption was made that a biallelic disease susceptibility gene with alleles $D$ and $d$ was uniformly located within the candidate region. The frequency of disease allele $D$ was set to be 0.1 . Let $R_{1}$ denote the relative risk with one copy of the disease allele $\mathrm{D}$ and $\mathrm{R}_{2}$ denote the relative risk with two copies compared with no copies. We considered a simple mutational process for the disease allele $D$ where the allele $D$ appears in an individual with the "ancestral haplotype" and it broke up because of meiotic recombination. We used the first-order Markov model [8] to generate the markers on the ancestral haplotype and then generate the haplotypes in the present-day population.

For the null hypothesis that neither association nor maternally mediated genetic effects exist, we let $R_{1}=R_{2}=1$. For the alternative hypothesis that there exists association or maternally mediated genetic effects, we considered the following three scenarios. First, there exists association where the state of disease phenotype of the offspring was determined by the offspring genotype; the second, there exists maternally mediated genetic effects where the state of disease phenotype of the offspring was determined by the mother genotype; and third, there exist both association and maternally mediated genetic effects where the state of disease phenotype of the offspring was determined by the genotypes of both mother and offspring. For the third scenario, we considered a simple model in which the relative risk was the product of the relative risks of the offspring and the mother. For the above three scenarios, we let the baseline disease risk (the penetrance of genotype $d d$ ) be 0.01 , and $R_{1}=R_{2}=2$ and $R_{1}=1, R_{2}=2$, which represented a dominant and a recessive model, respectively. The number of case-parent triads, $N$, was chosen as 100, 200, or 400. We assumed that each individual had an available marker that could be utilized as a genotype in the analysis. Upon simulation of the familial data, we first calculated the value of $\max _{-} P C$, and then recalculated it by using the permutation procedure 1,000 times. The $p$ value was the proportion of permutationbased statistics that were larger than the data-based statistic. We performed this data simulation with 10,000 replicates. For a given significance level $\alpha$, the power/type I error rate is then estimated as the proportion of rejecting the null hypothesis when $p \leq \alpha$.

We also investigated the performance of $\max _{-} P C$ in the presence of population admixture. We assumed that there were two subpopulations that differed in disease allele frequency and baseline disease risk. Disease allele $D$ was introduced 1,000 and 500 generations ago through two different ancestral haplotypes in the two subpopulations, respectively. The frequencies of the $D$ allele were set as 0.1 and 0.2 , and the baseline disease risks were set as 0.01 and 0.05 in the first and second subpopulation, respectively. We used the previously described approach to generate the markers and haplotypes in each subpopulation and let the two subpopulations admix at the last generation, with 30 percent for the first subpopulation and 70 percent for the second subpopulation.

\section{Assessment of the performance of the max_PC statistic}

As shown in Table 1, the type I error rates are around the nominal value, indicating that the type I error rates 
Table 1 Estimated type I error rates of the statistic, max_PC, for 10,000 simulations

\begin{tabular}{|c|c|c|c|c|c|c|}
\hline \multirow[b]{3}{*}{ Sample size } & \multicolumn{6}{|c|}{ Estimated type I error rates } \\
\hline & \multicolumn{3}{|c|}{ Homogeneous population } & \multicolumn{3}{|c|}{ Admixture population } \\
\hline & $a=0.05$ & $a=0.01$ & $a=0.001$ & $a=0.05$ & $a=0.01$ & $a=0.001$ \\
\hline 100 & 0.0486 & 0.0106 & 0.0012 & 0.0490 & 0.0095 & 0.0010 \\
\hline 200 & 0.0502 & 0.0092 & 0.0009 & 0.0487 & 0.0105 & 0.0009 \\
\hline 400 & 0.0489 & 0.0089 & 0.0018 & 0.0521 & 0.0110 & 0.0013 \\
\hline
\end{tabular}

are validated in both homogeneous and admixture populations. The powers under three scenarios are exhibited in Tables 2 and 3. The power of max_ $Z^{2}$ was also investigated so that we could compare the performance of max_PC with that of max_ $Z^{2}$. It should be noted that $\max \mathrm{Z}^{2}$ uses the difference vector $2 C-F-M$ and $M-F$ under the first scenario and the second scenario, respectively. However, we perform two tests under the third scenario: one uses the difference vector $2 C-F-M$ to calculate max_ $Z^{2}$ for testing association and the other uses the difference vector $M-F$ to calculate max_ $Z^{2}$ for testing maternally genetic effects. It is observed from Table 2 that, in homogeneous population, the power of max_PC for testing association and maternally mediated genetic effects is highest among three scenarios. The powers for testing association and maternally mediated genetic effects reach $80 \%$ and $90 \%$, respectively, when the sample size is 200 or 400 . The power of max_PC under the recessive model is slightly lower than those under the dominant mode. The second to the fifth columns of Table 2 show that the powers of max_PC are similar to those of max_ $Z^{2}$. Under the third scenario of testing association and maternally mediated genetic effects at the same time, the powers of $\max _{-} P C$ are slightly higher than those of max_ $Z^{2}$ with the difference vector $2 C-F-M$ and those of max_ $Z^{2}$ with $M-F$. Table 3 presents the powers in admixture population. It can be seen that the results are similar to those observed in homogeneous population.

\section{A real example}

To evaluate the performance of the max_PC on more realistic scenarios, we applied our method to a real data set of Crohn's disease. Crohn's disease is one of the two major forms of inflammatory bowel diseases [9, 10]. Rioux et al. $[10,11]$ detected a candidate region containing a genetic risk factor for Crohn disease on human chromosome 5q31with 139 case-parents trios. We applied the max_PC and the max_ $\mathrm{Z}^{2}$ to the publically available subset of 129 trios genotyped at 103 common SNPs. We assessed $P$ value using the permutation procedure 10,000 times. The $P$ value using the max_PC is $3.62 \times 10^{-4}$, indicating that this region is associated with Crohn's disease or there is a possible maternal effects. When the max_ $Z^{2}$ with $2 C-F-M$ and $M-F$ was used, the $P$ values are $3.78 \times 10^{-4}$ and $4.05 \times 10^{-3}$, respectively, indicating that there may exist association and maternal effects. In order to further compare the two methods, we also considered risk-haplotype-tagging alleles which are highly predictive of disease susceptibility as descripted by Shi et al. [4]. According to Shi et al. [4], we consider the locus with $P$ value being smaller than a preset threshold to be related to risk and designate the corresponding

Table 2 The powers of max_PC and max_ $Z^{2}$ for various tests based on simulations with global significance at $p \leq a(0.05)$ in a homogeneous population

\begin{tabular}{|c|c|c|c|c|c|c|c|}
\hline \multirow{2}{*}{$\begin{array}{l}\text { Test } \\
N=100\end{array}$} & \multicolumn{2}{|c|}{ Association } & \multicolumn{2}{|c|}{ Maternally mediated genetic effects } & \multicolumn{3}{|c|}{ Association and maternally mediated genetic effects } \\
\hline & max_PC & $\max z^{2}$ & max_PC & $\max \_z^{2}$ & max_PC & $\max \_z^{2 a}$ & $\max z^{2}$ b \\
\hline $\mathrm{R}_{1}=\mathrm{R}_{2}=2$ & 0.616 & 0.607 & 0.588 & 0.586 & 0.815 & 0.800 & 0.584 \\
\hline $\mathrm{R}_{1}=1, \mathrm{R}_{2}=2$ & 0.600 & 0.582 & 0.579 & 0.581 & 0.808 & 0.787 & 0.582 \\
\hline \multicolumn{8}{|l|}{$N=200$} \\
\hline$R_{1}=R_{2}=2$ & 0.819 & 0.820 & 0.725 & 0.711 & 0.917 & 0.902 & 0.713 \\
\hline$R_{1}=1, R_{2}=2$ & 0.803 & 0.810 & 0.702 & 0.704 & 0.911 & 0.898 & 0.702 \\
\hline \multicolumn{8}{|l|}{$N=400$} \\
\hline $\mathrm{R}_{1}=\mathrm{R}_{2}=2$ & 0.908 & 0.900 & 0.819 & 0.821 & 0.952 & 0.937 & 0.822 \\
\hline $\mathrm{R}_{1}=1, \mathrm{R}_{2}=2$ & 0.875 & 0.874 & 0.807 & 0.809 & 0.926 & 0.915 & 0.810 \\
\hline
\end{tabular}

${ }^{a}$ The max_ $Z^{2}$ using $2 C-F-M$

${ }^{\mathrm{b}}$ The max_Z $\mathrm{Z}^{2}$ using $M-F$ 
Table 3 The power of max_PC for various tests based on simulations with global significance at $p \leq a(0.05)$ in an admixture population

\begin{tabular}{|c|c|c|c|c|c|c|c|}
\hline \multirow{2}{*}{$\frac{\text { Test }}{N=100}$} & \multicolumn{2}{|c|}{ Association } & \multicolumn{2}{|c|}{ Maternally mediated genetic effects } & \multicolumn{3}{|c|}{ Association and maternally mediated genetic effects } \\
\hline & max_PC & max_Z2 & max_PC & max_z $Z^{2}$ & max_PC & $\max z^{2}$ a & $\max z^{2 b}$ \\
\hline$R_{1}=R_{2}=2$ & 0.602 & 0.600 & 0.564 & 0.561 & 0.803 & 0.787 & 0.562 \\
\hline$R_{1}=1, R_{2}=2$ & 0.587 & 0.576 & 0.551 & 0.548 & 0.788 & 0.769 & 0.547 \\
\hline \multicolumn{8}{|l|}{$N=200$} \\
\hline $\mathrm{R}_{1}=\mathrm{R}_{2}=2$ & 0.805 & 0.806 & 0.709 & 0.711 & 0.855 & 0.850 & 0.712 \\
\hline$R_{1}=1, R_{2}=2$ & 0.779 & 0.783 & 0.682 & 0.683 & 0.834 & 0.826 & 0.684 \\
\hline \multicolumn{8}{|l|}{$N=400$} \\
\hline$R_{1}=R_{2}=2$ & 0.878 & 0.879 & 0.800 & 0.798 & 0.882 & 0.875 & 0.796 \\
\hline$R_{1}=1, R_{2}=2$ & 0.864 & 0.867 & 0.776 & 0.777 & 0.879 & 0.870 & 0.769 \\
\hline
\end{tabular}

a The max_ $Z^{2}$ using $2 C-F-M$

bThe max_ $Z^{2}$ using $M-F$

overtransmitted allele as a risk-haplotype-tagging allele. It can be seen from Fig. 1 that the number of risk-haplotypetagging alleles identified using the $\max P C$ (Fig. 1c) is larger than that using the max_ $\mathrm{Z}^{2}$ (Fig. 1a and b).

\section{Discussion}

Based on the principal component analysis, we proposed a statistic max_PC to test association and maternally mediated genetic effects with multiple markers. Similar to the max_ $Z^{2}$ test, the max_PC test only uses the genotypes of case-parent triads and accommodates missing SNP data. Notably, at least two characteristics of the max_ $P C$ test are totally different from those of the max_ $Z^{2}$ test: (1) the $\max _{-} P C$ test uses the principal component analysis, and (2) the max_PC can be used to test association and maternally mediated genetic effects at the same time, whereas two tests with max_ $\mathrm{Z}^{2}$ should be performed for this case. The performance of max_PC was assessed by simulations analysis as well as the application in Crohn's disease study.

In practice, when multiple markers are studied, individuals may have incomplete information of individual marker data. Our method calculates the $P C$ value for each locus, indicating that it is capable of handling missing SNP data. Moreover, our approach is not biased toward admixture population and Hardy-Weinberg equilibrium was not essential when using our method. The null hypothesis for the max_PC test is that there is no association and no maternally mediated genetic effects, whereas the alternative hypothesis is that there is an association or maternally mediated genetic effects. One attractive feature of $\max \_P C$ is that it is able to test association and maternally mediated genetic effects exist at the same time. Also, it provides a method for testing the null hypothesis of no association and no maternally mediated genetic effects. When the null hypothesis is rejected we will use max_ $Z^{2}$ with the difference vector, $2 C-F-M$, for testing association, and use max_ $\mathrm{Z}^{2}$ with the difference vector, $M-F$, for testing maternally mediated genetic effects. In this way, we will be able to determine whether only maternally mediated genetic effects or only association exists. It is worth noting that testing for maternally mediated genetic effects rely on the assumption of mating symmetry when max_PC was used according to $M-F$. However, when an offspring effect is present, parent-of-origin effects can also cause the effect of symmetry [4]. In this case, it is not easy to distinguish maternally mediated genetic effects from parent-of-origin effects. Further studies to carefully evaluate the possible parent-of-origin effects are thus warranted.

\section{Conclusions}

We have proposed a statistical approach for detecting association or maternally mediated genetic effects with case-parent data. The approach is powerful to test association and the maternally mediated genetic effects at the same time. It also provides a valid tool for testing no association and no maternally mediated genetic effects. The proposed method for association analysis incorporating maternally mediated genetic effects is particularly useful in studying diseases that happened during fetal life.

\section{Methods}

In this study, all datasets were publically available and no research requiring ethics approval was conducted.

We consider a biallelic marker with alleles "A" and "a". Assume that case-parent triads are sampled with the genotypes known for each member of the triads. Let $M, F$, and $C$ be the number of copies of allele "A" carried by the mother, father, and affected offspring, respectively. $2 C-F-M$ is the paired differences in genotypes between the affected offspring and the complement, which carries the non-transmitted genotypes in the case-parent data. $M-F$ is the SNP-count difference between the mother and the father, which can be used to measure 

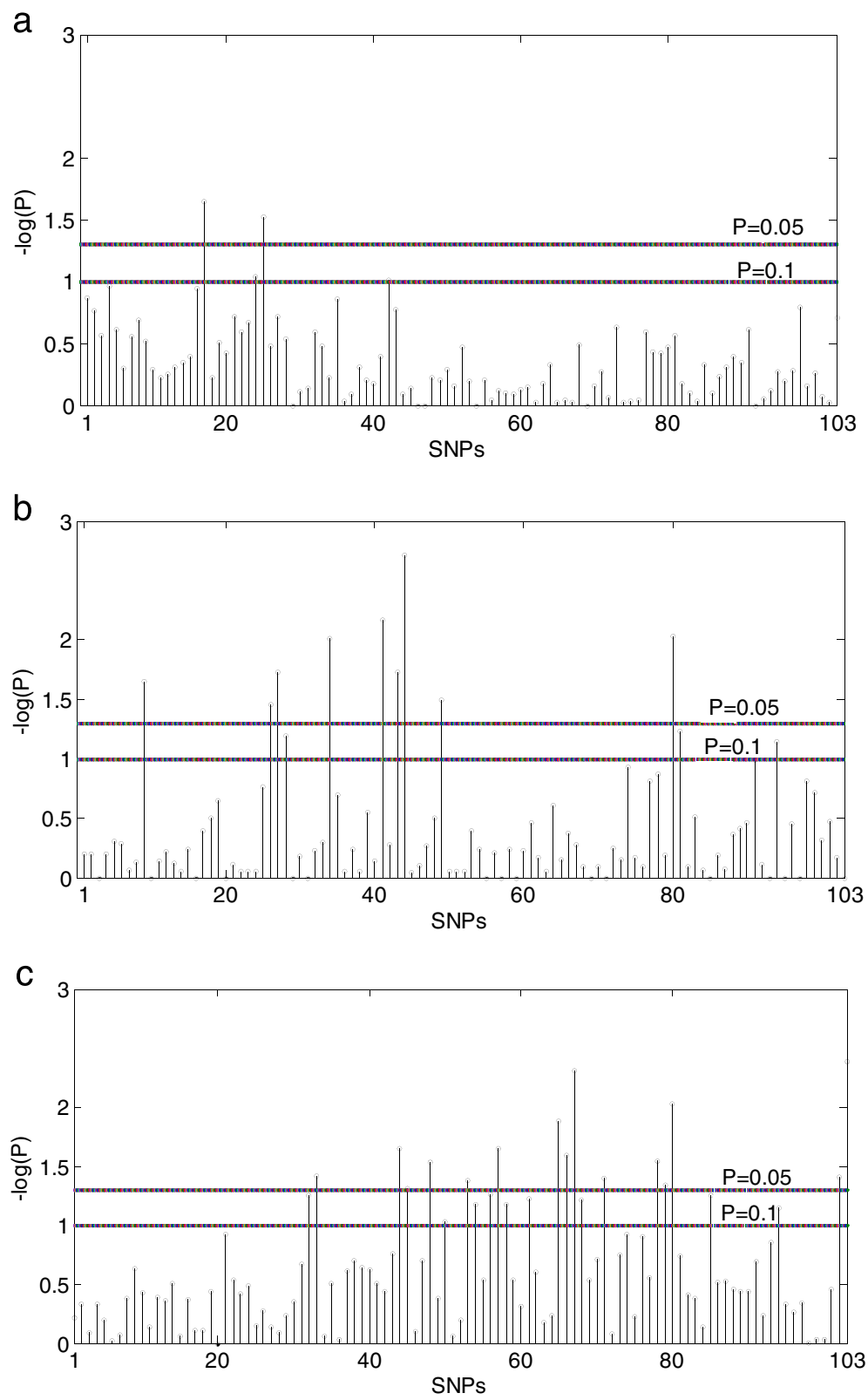

Fig. 1 Crohn's disease study. Result of testing association using the max_ $Z^{2}$ with $M-F(\mathbf{a})$, testing maternal effects using the max_ $Z^{2}$ with $2 C-F-M(\mathbf{b})$, and testing association or maternal effects using the max_PC (c). The $Y$-axis shows $-\log 10(p)$ at individual SNPs; the $X$-axis shows SNPS

the maternally mediated genetic effects with the assumption of mating symmetry. Let $X=2 C-F-M$ and $Y=M-F . X=0$ when there is no association, and $Y=0$ when there is no maternally mediated genetic effects. Define a two-dimensional random variable $Z=(X, Y)^{T}$. Under the null hypothesis of no association and no maternally mediated genetic effects, $Z=0$. We assume that there are $n$ case-parent triads. Let $S$ be the sample covariance matrix of $Z$ for the observed case-parent trios data, $Z_{i}=\left(X_{i}, Y_{i}\right)^{T}(i=1, \cdots, n)$. Denote the two sample eigenvalue-eigenvector pairs for $S$ by $\left(\lambda_{1}, e_{1}\right),\left(\lambda_{2}, e_{2}\right)$, where $\lambda_{1} \geq \lambda_{2} \geq 0$. Then, the $k$ th $(k=1,2)$ sample principal component is $\zeta_{k}=\hat{e}_{k}^{T} Z$, with variance of $\operatorname{Var}\left(\zeta_{k}\right)=\hat{\lambda}_{k}$. Note that the two sample principal components $\zeta_{1}, \zeta_{2}$ are uncorrelated, i.e., $\operatorname{Cov}\left(\zeta_{1}, \zeta_{2}\right)=0$. The $k$ th sample principal component for the $i$ th case-parent trio is $\zeta_{i k}=\hat{e}_{k}^{T} Z_{i}(k=1,2 ; i=1, \cdots, n)$. Let $\bar{\zeta}_{k}=\frac{1}{n} \sum_{i=1}^{n} \zeta_{i k}$. Here, $E\left(\bar{\zeta}_{k}\right)=E\left(\zeta_{k}\right), \quad \operatorname{Var}\left(\bar{\zeta}_{k}\right)=\frac{1}{n} \hat{\lambda}_{k}$, and $\operatorname{Cov}\left(\bar{\zeta}_{1}, \bar{\zeta}_{2}\right)=0$ $(k=1,2)$. Under the null hypothesis of no association 
and no maternally mediated genetic effects, $E\left(\bar{\zeta}_{k}\right)=0$ for $k=1,2$. Define a statistic, denoted by $P C$, as follow:

$$
P C=\frac{n\left(\sum_{k=1}^{2} \bar{\zeta}_{k}\right)^{2}}{\sum_{k=1}^{2} \hat{\lambda}_{k}}
$$

The statistic $P C$ is asymptotically a central $\chi_{(1)}^{2}$ distribution under the null hypothesis of no association and no maternally mediated genetic effects.

Now, we consider $q$ biallelic markers each with alleles "A" and "a". The markers are indexed by $l(l=1, \cdots, q)$. Let $P C^{(l)}$ be the statistic for marker $l$. Our test statistic, here, denoted as max_PC, is the maximum of the $P C^{(l)}$ across all $q$ marker loci, i.e., $\max -P C=\max _{l}\left\{P C^{(l)}\right\}$. It can be seen that this max_PC approach is able to accommodate missing individual marker genotypes and does not require any haplotype information. Here, the statistical significance is assessed by a permutation procedure. We first calculate the data-based statistic. Then we permute the "case" and "complement" labels with equal probability and recalculate the statistic. The estimated $P$ value (denoted by $\hat{p}$ ) is then the proportion of permutation-based statistics that are larger than the data-based statistic.

\section{Availability of supporting data}

The data set used in this article is shown in Tables 1, 2 and 3. Data of Crohn's disease are available from http:// www.broadinstitute.org/archive/humgen/IBD5/raw_data.txt and http://www.broadinstitute.org/archive/humgen/IBD5/ haplodata.html.

\section{Competing interests}

The authors declare that they have no competing interests.

\section{Authors' contributions}

LYM conceived the idea, designed the study, and wrote the manuscript. XY developed the statistical method. Both authors have read and approved the final version of the manuscript.

\section{Acknowledgements}

The study was supported by National Natural Science Foundation of China (11301206).

Received: 9 September 2015 Accepted: 14 January 2016

Published online: 19 January 2016

\section{References}

1. McIntyre LM, Martin ER, Simonsen KL, Kaplan NL. Circumventing multiple testing: a multilocus Monte Carlo approach to testing for association. Genet Epidemiol. 2000;19(1):18-29.

2. Lee WC. Testing for candidate gene linkage disequilibrium using a dense array of single nucleotide polymorphisms in case-parents studies. Epidemiology. 2002;13(5):545-51.

3. Fan R, Knapp M, Wjst M, Zhao C, Xiong M. High resolution T2 association tests of complex diseases based on family data. Ann Hum Genet. 2005;69(2):187-208.

4. Shi M, Umbach DM, Weinberg CR. Identification of risk-related haplotypes with the use of multiple SNPs from nuclear families. Am J Hum Genet. 2007:81(1):53-66.
5. Han $M, H u Y Q$, Lin SL. Joint detection of association, imprinting and maternal effects using all children and their parents. Eur J Hum Genet. 2013;21(12):1449-56.

6. Wilcox AJ, Weinberg CR, Lie RT. Distinguishing the effects of maternal and offspring genes through studies of "case-parent triads". Am J Epidemiol. 1998;148(9):893-901.

7. Devlin B, Risch N. A comparison of linkage disequilibrium measures for fine-scale mapping. Genomics. 1995;29(2):311-22.

8. McPeek MS, Strahs A. Assessment of linkage disequilibrium by the decay of haplotype sharing, with application to fine-scale genetic mapping. Am J Hum Genet. 1999;65(3):858-75.

9. Daly MJ, Rioux JD, Schaffner SF, Hudson TJ, Lander ES. High resolution haplotype structure in the human genome. Nat Genet. 2001;29(2):229-32.

10. Rioux JD, Daly MJ, Silverberg MS, Lindblad K, Steinhart H, Cohen Z, et al. Genetic variation in the $5 q 31$ cytokine gene cluster confers susceptibility to Crohn disease. Nat Genet. 2001;29(2):223-8.

11. Rioux JD, Silverberg MS, Daly MJ, Steinhart AH, McLeod RS, Griffiths AM, et al. Genomewide search in Canadian families with inflammatory bowel disease reveals two novel susceptibility loci. Am J Hum Genet. 2000;66(6):1863-70

Submit your next manuscript to BioMed Central and we will help you at every step:

- We accept pre-submission inquiries

- Our selector tool helps you to find the most relevant journal

- We provide round the clock customer support

- Convenient online submission

- Thorough peer review

- Inclusion in PubMed and all major indexing services

- Maximum visibility for your research

Submit your manuscript at www.biomedcentral.com/submit
) Biomed Central 